\title{
Efficient Voting with Penalties
}

\author{
Maksymilian Kwiek*
}

May 26, 2017

\begin{abstract}
Simple majority does not reflect the intensity of voters' preferences. This paper presents an efficient collective choice mechanism with two alternatives when the designer may use non-trasferable punishments to persuade agents to reveal their private information. The designer faces a dilemma - a punishment may induce a more correct choice, but its cost is socially wasteful. The efficient mechanism is a weighted majority. Weight of each individual is known ex ante and no punishments are applied if preferences are relatively homogeneous. Eliciting types through punishments in order to construct type-specific weights should occur if preference intensity is relatively heterogeneous.
\end{abstract}

JEL classification: D71, D82

Keywords: Voting, mechanism design

\section{Introduction}

Simple majority is the benchmark of voting systems. The seminal contribution of Rae (1969) indicates that when choice is binary, simple majority has good normative properties. The key assumption in Rae (1969) is that the intensity of voters' preferences in favor of an alternative is always the same. The present paper revisits the question of normative performance of various voting systems under the assumption that voters may differ in the intensity of preferences over two possible alternatives, and that interpersonal comparisons are possible.

As in many voting mechanisms, monetary transfers are not allowed. The main premise is that voters may be punished, and thus punishments can be a part of the mechanism's design. A benevolent designer faces a dilemma -

\footnotetext{
*Department of Economics, University of Southampton, SO17 1BJ, UK, M.Kwiek@soton.ac.uk
} 
punishments can motivate voters to reveal the intensity of their preferences, facilitating a correct assignment, but at the same time they represent an unrecoverable welfare loss.

There are real-life examples of collective choice mechanisms in which preference intensity can be expressed in a wasteful way. Probably the most straightforward case is majority voting with a lump-sum cost of participation - only voters whose stakes are high are likely to take part. As long as the cost of participation is socially wasted, this procedure belongs to the class of mechanisms studied here. Another example is repeated voting with a supermajority - voters are locked until the support for one of the alternatives reaches some supermajority, or the support for the other one falls below some minimal level. A form of a war of attrition ensues, in which waiting time imposes a cost on participating voters, and this cost is a pure welfare loss. A conclave with supermajority $2 / 3$ is used to select a Pope - the leader of the Roman Catholic Church. Many hiring committees vote repeatedly until one of the candidates gathers enough support. Jury trials use a similar procedure of repeated voting until all jurors agree on a verdict. Yet another example is lobbying to influence a binary political decision: non-cooperative lobbying efforts, wasteful as such, translate into a higher probability of winning.

In these real-life mechanisms and in an infinite number of non-existing but possible ones, specific design features could influence the outcomes in subtle and multifaceted ways. However, the current paper attempts to abstract from procedural details. Instead, it applies the techniques of mechanism design to characterize general features of an efficient mechanism when the mechanism designer is free to use penalties. This is not to say that specific procedures should not be studied by direct modeling - to the contrary, there are limits to what the current analysis can achieve. However, the efficiency bound derived from general incentive constrains provides a useful benchmark on welfare in any specific voting scheme.

\section{A brief summary of the results}

Voters' preferences, called types, are their private information. The main result is that regardless of whether the voting mechanism should seek to reveal and use voters' preference types, an efficient mechanism always takes a form of weighted majority. The main dilemma faced by the designer revolves around the question of whether these weights should be type-dependent, and thus whether voters should be incentivized to reveal their types.

The solution to this dilemma depends on the level of dispersion, or heterogeneity in voters' preferences. At this stage, it is useful to distinguish between the ex post heterogeneity, which refers to the dispersion of the real- 
ized preferences, and the ex ante heterogeneity, which is concerned with how likely those randomly chosen preferences exhibit high dispersion. For example, if the distribution of intensities is non-degenerate, then there is some ex ante heterogeneity, but the actually realized preferences may be dispersed or not ex post.

The level of ex ante heterogeneity in preferences is the key determinant of whether extracting information from the voters is desirable. If intensity of preferences is not ex ante heterogeneous enough, then improving the likelihood of selecting a better alternative is not worth the associated social cost of screening. Consequently, the efficient mechanism is a weighted majority with weights known ex ante. It does not extract any information and does not apply any penalties. In addition to this, if the environment is symmetric, then these weights are equal, and the mechanism is the old-fashioned simple majority; Rae (1969) can be viewed as the polar case of no ex ante heterogeneity.

On the other hand, if intensity of preferences is ex ante heterogeneous enough, the efficient mechanism extracts information from the voters and uses it to construct type-dependent weights. Penalties are needed to create incentives for the voters to reveal their types. A simple mechanism implementing the efficient outcome in dominant strategies is easy to characterize. The real-life examples of voting mechanisms, such as a referendum with participation cost, multi-player war of attrition or lobbying are generally not efficient, although they may generate a higher welfare than simple majority.

This paper also explores the role of ex post realization of preferences. Firstly, in any efficient mechanism more agents supporting one alternative weakly increases the chances of selecting this alternative. This is a strong result as it does not depend on any extra conditions. Secondly, if the reciprocal hazard rate is increasing, so that ex ante heterogeneity is high enough for the preference intensity to matter, then higher preference intensity leads to weakly higher chances of this alternative being selected. Thirdly, if the reciprocal hazard rate is increasing, then ex post heterogeneity of preferences also matters, but the relationship is difficult to capture intuitively as it depends on the curvature of the reciprocal hazard rate. Having voters with more dispersed realized preferences supporting one alternative weakly increases the likelihood of selecting their alternative, if the reciprocal hazard rate is convex. Having voters with less dispersed realized preferences supporting one alternative increases the likelihood of selecting their alternative, if the reciprocal hazard rate is concave. 


\section{Broader context and objectives}

This paper explores a link between two separate strands of literature on efficient social choice with privately known preferences: one discussing environments permitting transfers, and one where transfers are not allowed. Secondly, the efficient benchmark proposed in this paper and the techniques adopted from auction theory may be useful to those researchers who analyze real-life voting methods. In the remainder of this section, these two objectives are discussed in the context of the received literature; some assumptions and further results are also examined.

Desirable social outcomes might be achieved if privately known preferences of the agents are revealed and properly integrated into the social choice problem. This can happen only if agents have correct incentives - often framed as taxes or subsidies - to truthfully reveal the intensity of their preferences. There are many mechanisms that lead to allocative efficiency, for example, the famous $V C G$ mechanism achieves this in dominant strategies (Vickrey (1961), Clarke (1971), Groves (1973)). ${ }^{1}$

As it turns out, the $V C G$ mechanism necessarily generates a positive net tax revenue. This fact causes a small dilemma - any Pareto efficient outcome should not waste this revenue, but if it is returned to the participants, then the mechanism's desirable welfare properties are destroyed (Tideman and Tullock (1976), Groves and Ledyard (1977)). One possible solution is to confiscate the revenue and transfer it to some external agents who have no stakes in the outcome of the mechanism. The other possibility is to waste this revenue and hope that its magnitude is not significant (Tideman and Tullock (1976), Rob (1982)). However, if the mechanism cannot be redeemed in either of these two ways, then the relevant welfare question has to be modified into the following: what is the efficient dominance-solvable mechanism if the tax revenue is to be wasted? This is one way to frame the main question asked in the present paper. ${ }^{2}$

The alternative viewpoint on collective choice problems is that monetary taxes and subsidies are not allowed. The literature on why transfers may

\footnotetext{
${ }^{1}$ In its simplest form, this mechanism asks the participants to reveal their valuations, and then selects the alternative that generates the greatest total value. Revealing true valuations is a dominant strategy because of the clever way participant's payment is set: it is equal to the net externality caused by this participant on other agents - it depends only on those other agents' reports.

${ }^{2}$ If only Bayesian incentive compatibility is required rather than dominance solvability, then there exists a budget balanced mechanism which implements an efficient outcome. It is known as the expected externality mechanism (or AGV mechanism); see Arrow (1979), d'Aspremont and Gérard-Varet (1979). A newer study in a similar vein is, for example, Goeree and Zhang (2013).
} 
be ruled out in the first place is not very large. For example, in the context of public good provision Ledyard and Palfrey (1994), (1999) and (2002) suggest that agents' budget constraints, welfare distribution concerns, or a complexity of a payment mechanism may be possible explanations. Most of the voting literature simply commits to ordinal preferences framework and rules out transfers a priori. What this approach misses is that screening is possible even without monetary payments. Waiting, lobbying and other costs of political action satisfy the axiom of no monetary transfers, but may replace monetary taxes as an incentive device. ${ }^{3}$

The literature on efficiency of mechanisms not using transfers starts with the aforementioned paper by Rae (1969); see also May (1952). A modern version of this question, in which private information takes center stage, gathered some attention recently; ${ }^{4}$ the most relevant to the present study is Azrieli and Kim (2014). They ask what mechanism is efficient in the binary choice environment when transfers or penalties are not available. Their answer is weighted majority. The present paper asks the same question, but it allows the designer to use non-transferable penalties. This is a significant modification, but the answer is markedly similar to Azrieli and Kim (2014), at least in some cases. The efficient mechanism is weighted majority, although - as stated in the previous section - the issue is whether penalties should be applied and private information extracted in order to calculate these weights. If the answer is negative, then an argument in favor of simple majority is provided, and the mechanism is exactly like the one in Azrieli and $\operatorname{Kim}(2014) .{ }^{5}$ On the other hand, it is also shown when simple majority might be improved upon by using meaningful penalties.

The second objective of this study is to propose a way of analyzing real-life multiplayer decision mechanisms with penalties. There is a small literature that investigates their positive or normative properties, although in all but the simplest cases the direct analysis of equilibria is difficult. ${ }^{6}$ The current

\footnotetext{
${ }^{3}$ There may be good reasons why non-monetary penalties are difficult to use, in exactly the same way as is the case for monetary transfers. These reasons should be investigated explicitly in the relevant application.

${ }^{4}$ For example, Schmitz and Tröger (2012) consider correlated signals, while Apesteguia at al. (2011) and Gershkov at al. (2014) analyze the case of more than two alternatives.

${ }^{5}$ Drexl and Kleiner (2013) and Kwiek and Zhang (2013) introduce a similar model where voters's payments are wasted, but they focus on the case of low ex ante heterogeneity, thus characterizing the efficient mechanism as weighted majority without any penalties.

${ }^{6}$ Ponsati and Sakovics (1996) are concerned with properties of equilibria in multiplayer Wars of Attrition with supermajority. Kwiek (2014) is focused on welfare performance of these kind of mechanisms, when the cost of penalty does not enter the welfare function. Kwiek et al. (2016) experimentally study positive and welfare properties of similar waiting games.
} 
paper suggests that techniques from auction theory may help to shed some light on the performance of such mechanisms.

To be more precise, the analysis given in the present paper draws heavily from the Myerson's (1981) seminal characterization of incentive-compatible mechanisms in the context of auctions. McAfee and McMillan (1992), followed by a series of more recent papers by Hartline and Roughgarden (2008), Yoon (2011), Condorelli (2012) and Chakravarty and Kaplan (2013), use a similar approach to provide normative analysis when participants' bids or efforts are a social waste, rather than the auctioneer's revenue. The key object in this analysis is the allocation function that maps the profile of individual preferences into the likelihood of selecting one of the alternatives. If a particular mechanism can be framed in terms of such equilibrium allocation function (a big if), then welfare comparative statics becomes tractable.

While using essentially the same technique as Myerson's (1981), McAfee and McMillan (1992) and others, the current paper innovates in a few ways. The key difference is that this literature looks at an assignment problem how to allocate a rivalrous good among competing individuals. In contrast, the present study considers a collective choice problem, giving rise to different forms of externalities among the agents. For example, in the spirit of the classical Samuelson condition for public goods (Samuelson, (1954)), the efficiency condition involves the sum of individual benefits over all agents supporting an alternative. However, these individual benefits are net of information revelation costs, as in the tradition of Myerson's (1981) and McAfee and McMillan (1992).

Another difference is that in the model given in this paper, private information is multidimensional. Apart from the usual preference intensity, individual voters are assumed to have private information about the cost of punishment. $^{7}$ This extra layer of private information is quite important in the context of the economic environment studied in the present paper, because penalties are not interpreted necessarily as monetary payments, and thus the marginal cost does not have to be equal to one, or even commonly known. Once it is recognized that imposing wasteful efforts is available in the designer's tool-box, one may contemplate very exotic and completely artificial punishments such as subjective or monetary cost of waiting, tedious tasks, electric shocks, etc. Selecting a type of penalty becomes a nontrivial question, and the precise form of statistical dependence between the intensity of preferences and marginal cost of penalty plays a role. Even if using

\footnotetext{
${ }^{7}$ Chakravarty and Kaplan (2013) also assume that agents may differ in their individual cost parameters of effort, but here I assume that the value and the cost parameter are two different random variables, possibly statistically independent.
} 
one type of penalty leads to the efficient mechanism being simple majority without penalties, having another type may make it worthwhile to extract private information. Technically, the environment is still relatively tractable, the incentive compatibility can be framed as the usual integrability condition a la Myerson (1981), and the complications of multidimensional types, such as the ones in Armstrong (1996), do not arise.

A few final remarks are in order. There is a different category of recent literature investigating how preference intensities can be reflected in voting mechanisms. Casella (2005) proposes a procedure in which agents can store their votes for future use in a sequence of elections, thus creating an opportunity to deploy accumulated votes on issues of a particular importance. Casella et al. (2006) experimentally investigate this idea. A similar concept of linking different social choice problems to enhance incentives appears in Jackson and Sonnenschein (2007). Casella and Gelman (2008) apply the linkage idea to bundled referenda. In contrast to this line of research, the present study assumes a single social choice problem.

This paper claims that, even in binary agenda case, complicated voting rules may be justifiable, because they may allow voters to express the intensity of their preferences. However, there are other reasons why complicated voting rules may be beneficial. For example, committees may be viewed as a tool for revealing correlated information - the model presented in the current paper assumes that agents' private information is statistically independent across individuals, so this type of question cannot be asked. Moreover, the aim of complicated rules may be to incentivize efforts to learn the relevant information - the current paper does not tackle a moral hazard problem of this type.

Section 2 introduces the model. Section 3 presents the mechanism design problem and the incentive compatibility characterization. Section 4 is the core part establishing the main result in Theorem 1 . Section 5 discusses comparative statics, in particular the role of ex ante heterogeneity of preference intensity and the question of selecting the type of penalty. Section 6 illustrates the ideas of this paper with an example of a voting game with entry cost - a realistic but non-efficient mechanism. The last Section concludes.

\section{Environment and welfare criteria}

Physical environment consist of the following elements. One alternative is to be selected from the set $\left\{B_{1}, B_{2}\right\}$, and $k$ will denote a generic alternative. There are $n \geq 2$ voters (participants, committee members, agents). The key postulate is that there exists a way to penalize individuals. Namely, 
assume that an individual voter $i$ can be forced to suffer $c_{i}$ units of a penalty. One may hypothesize that there are different types of penalties, and the mechanism designer may have an option to select one. For now the penalty type is fixed, but this assumption will be revisited later.

Preferences of each voter are characterized by three components. The first element, $a_{i} \in\left\{B_{1}, B_{2}\right\}$, is called voter's location and describes which alternative voter $i$ prefers. Sometimes we will refer to the set of players supporting $k$ as party $k$. The second element describes the intensity of this preference. Intensity is denoted by $x_{i} \geq 0$, interpreted as how many units of penalty this voter is willing to endure at most, and still select her preferred alternative over the other alternative if it is received without penalty. The third element is $z_{i}$, the cost parameter affecting the cost of penalty to individual $i$.

The ultimate net payoff of voter $i$ who is asked to suffer $c_{i}$ units of penalty is

$$
\begin{cases}z_{i}\left(x_{i}-c_{i}\right) & \text { if her preferred alternative is selected } \\ -z_{i} c_{i} & \text { otherwise }\end{cases}
$$

Notice that the top line can also be written more conventionally as $v_{i}-z_{i} c_{i}$, where $v_{i}=z_{i} x_{i}$ could be called the absolute intensity of preferences, since its magnitude is independent of the penalty type (in contrast to relative intensity $x_{i}$, which is defined in terms of penalty). As it will become clear later, $x_{i}$ and $z_{i}$ will be values of some random variables which may or may not be statistically independent. ${ }^{8}$

The final element that has to be specified in order to complete the description of the model is the information available to the voters and to the mechanism designer. Assume that all three elements of voter's preferences are her private information. That is, voter $i$ knows the realization of the triplet of random variables $\left(A_{i}, X_{i}, Z_{i}\right)$, denoted $\left(a_{i}, x_{i}, z_{i}\right)$, and called the type of voter $i$. Other voters and the mechanism designer know only its joint distribution.

Types are independent across individuals. The intensity and cost parameter might not be independent for a given individual; we assume that the p.d.f. and c.d.f. of $X_{i}$ conditional on $Z_{i}$ is $f_{i}\left(x_{i} \mid z_{i}\right)$ and $F_{i}\left(x_{i} \mid z_{i}\right)$, while the

\footnotetext{
${ }^{8}$ In this formulation, the cost parameter $z_{i}$ is simply the marginal cost of penalty for player $i$, constant with respect to the quantity of penalty. A more general formulation would assume that the cost is $z_{i} c_{i}\left(s_{i}\right)$, where the physical quantity of penalty $s_{i}$ may affect the perception of penalty through a function $c_{i}(\cdot)$, not necessarily linear or even increasing (but whose image is $R_{+}$). In the current analysis, the only thing that matters is penalty measured in utils, so there is no generality loss in assuming that the penalty cost is simply $z_{i} c_{i}$ instead. However, to emphasize that we are not assuming that marginal cost is constant, $z_{i}$ will be called the cost parameter rather than marginal cost.
} 
marginal p.d.f. of $X_{i}$ is $f_{X_{i}}\left(x_{i}\right)$. For any $z_{i}$, function $F_{i}\left(x_{i} \mid z_{i}\right)$ is continuous and strictly increasing for $x_{i} \in\left(0, x_{i}^{\prime}\right)$, where $x_{i}^{\prime}$ could be infinity. It is assumed that $A_{i}$ and $\left(X_{i}, Z_{i}\right)$ are statistically independent, but this is only for transparency of notation. It is also assumed that the expected value of $v_{i}$ conditional on $x_{i}$ is increasing in $x_{i}$.

This paper adopts a utilitarian welfare criterion as a benchmark capturing interpersonal comparisons of intensity of preferences. That is, social net welfare is the sum of individual payoffs and it is equal to

$$
\sum_{i: a_{i}=B_{k}} v_{i}-\sum_{i=1}^{n} z_{i} c_{i}
$$

if alternative $k$ is selected, and a vector of penalties $c_{1}, \ldots, c_{n}$ is applied.

This notion takes into account the costs of voting suffered by the participants. Later, we will observe that there is no difficulty in investigating allocative welfare, where the cost of penalties is ignored in welfare calculation, or any convex combination of the two types of efficiency. Likewise, checking any point on the ex ante expected Pareto Frontier is possible too.

Since the cost parameter is just a constant in front of the payoff function, it may appear that it will not play any role in the analysis, except perhaps in scaling the payoffs. Indeed, the presence of a privately known $z_{i}$ will pose no major complication in deriving the incentive compatibility condition in the next section, or in the general characterization of the efficient mechanism in Section 4. However, comparative statics results will be discussed in some detail in Section 5, where the stochastic relationship between random variables $Z_{i}, X_{i}$ and $V_{i}=X_{i} Z_{i}$ will turn out to play an important role.

\section{Incentive compatible mechanisms}

The mechanism designer specifies a Bayesian game, in which the voters play a Bayesian Nash equilibrium. By revelation principle any mechanism can be mimicked by a direct revelation and incentive compatible mechanism. In such a mechanism each player simultaneously reports their type, having incentives to report truthfully, and then the mechanism executes the outcome.

Let $a=\left(a_{1}, \ldots, a_{n}\right)$ be the profile of true preference locations, let $x=$ $\left(x_{1}, \ldots, x_{n}\right)$ be the profile of true intensities and $z=\left(z_{1}, \ldots, z_{n}\right)$ the profile of true cost parameters. Let $\bar{a}=\left(\bar{a}_{1}, \ldots, \bar{a}_{n}\right)$ be the profile of reported preference locations, and let $\bar{x}$ and $\bar{z}$ be similar vectors of reported intensities and cost parameters. Reports may be different from true values. To shorten the notation, we will write $r_{i}=\left(a_{i}, x_{i}, z_{i}\right)$; likewise for reports and profiles: $\bar{r}_{i}=$ $\left(\bar{a}_{i}, \bar{x}_{i}, \bar{z}_{i}\right), r=(a, x, z), \bar{r}=(\bar{a}, \bar{x}, \bar{z})$. 
The direct revelation mechanism is a collection $\left(p_{B_{1}}, p_{B_{2}}, c_{1}, \ldots, c_{n}\right)$, where all elements are functions of the report profile $\bar{r}$. The mechanism works as follows. After the agents have reported their preferences to the mechanism, alternative $k$ is eventually selected with probability $p_{k}(\bar{r}) \in[0,1]$, where obviously $p_{B_{1}}(\bar{r})+p_{B_{2}}(\bar{r})=1$. In this notation, $p_{a_{i}}(\cdot)$ is the probability with which the alternative that voter $i$ prefers is selected. Then, agent $i$ has to pay a non-negative and non-transferable expected penalty, $c_{i}(\bar{r}) \geq 0$. The expected payoff of voter $i$, whose preference type is $r_{i}$ while the report profile of types was $\bar{r}$, is

$$
z_{i}\left(x_{i} p_{a_{i}}(\bar{r})-c_{i}(\bar{r})\right)
$$

Define $P_{a_{i}}\left(\bar{r}_{i}\right)=E_{r_{-i}} p_{a_{i}}\left(\bar{r}_{i}, r_{-i}\right)$ to be the expected probability of $i$ 's favorite alternative being selected if her report is $\bar{r}_{i}$ (not necessarily truthful) when all other voters report truthfully, and $r_{-i}=\left(r_{1}, \ldots, r_{i-1}, r_{i+1}, \ldots, r_{n}\right)$; define $C_{i}\left(\bar{r}_{i}\right)=E_{r_{-i}} c_{i}\left(\bar{r}_{i}, r_{-i}\right)$ to be the expected penalty in this situation; finally define

$$
\tilde{\pi}_{i}\left(\bar{r}_{i}, r_{i}\right)=x_{i} P_{a_{i}}\left(\bar{r}_{i}\right)-C_{i}\left(\bar{r}_{i}\right)
$$

and

$$
\pi_{i}\left(r_{i}\right)=\tilde{\pi}_{i}\left(r_{i}, r_{i}\right)
$$

Clearly, the expected payoff of voter $i$, if all voters other than $i$ report truthfully, is simply $z_{i} \tilde{\pi}_{i}\left(\bar{r}_{i}, r_{i}\right)$, and the expected payoff when voter $i$ reports truthfully along with everyone else is $z_{i} \pi_{i}\left(r_{i}\right)$.

Proposition 1. The direct revelation mechanism is incentive compatible if and only if all conditions hold:

1. $P_{a_{i}}\left(a_{i}, \cdot, z_{i}\right)$ is non-decreasing

2. $\frac{\partial}{\partial x_{i}} \pi_{i}\left(a_{i}, x_{i}, z_{i}\right)=P_{a_{i}}\left(a_{i}, x_{i}, z_{i}\right)$

3. $P_{a_{i}}\left(a_{i}, 0, z_{i}\right) \geq P_{a_{i}}\left(-a_{i}, 0, z_{i}\right)$ and $C_{i}\left(a_{i}, 0, z_{i}\right)=C_{i}\left(-a_{i}, 0, z_{i}\right)$

4. $\pi_{i}\left(a_{i}, x_{i}, \cdot\right)$ and $P_{a_{i}}\left(a_{i}, x_{i}, \cdot\right)$ are constant functions.

The proof is in the Appendix. Conditions 1 and 2 of this Proposition ensure that individual $i$ does not have incentives to misreport her intensity $x_{i}$, and they are standard in mechanism design literature. ${ }^{9}$ Condition 3 makes certain that voters have no incentives to misreport their locations. Condition 4 guarantees that voters will not lie about their cost parameter. This

\footnotetext{
${ }^{9}$ For example, see Lemma 2 in Myerson (1981), or Proposition 23.D.2 in Mas-Colell et al. (1995).
} 
simplifies the notation, so that we can write $\pi_{i}\left(a_{i}, x_{i}\right), P_{a_{i}}\left(a_{i}, x_{i}\right), C_{i}\left(a_{i}, x_{i}\right)$ and $p_{a_{i}}(a, x)$.

Before moving on, let us observe that if the mechanism does not use reported intensities to select the alternative, like for example in case of simple majority, then the expected penalty applied to the agents cannot vary with the reported intensities either. This follows from condition 2 of the Proposition; if the function $P_{a_{i}}\left(a_{i}, \cdot\right)$ is constant, so is $C_{i}\left(a_{i}, \cdot\right)$.

\section{Welfare maximizing mechanisms}

It turns out that, as in McAfee and McMillan (1992), the core criterion for net efficiency involves the reciprocal hazard rate associated with the distribution of $x_{i}$. Specifically, let $H_{i}(\cdot)$ be defined as

$$
H_{i}\left(x_{i}\right)=E_{Z_{i}} z_{i} \frac{1-F_{i}\left(x_{i} \mid z_{i}\right)}{f_{X_{i}}\left(x_{i}\right)} .
$$

For example, if $z_{i}$ and $x_{i}$ are statistically independent, then $H_{i}(\cdot)$ is the conventional reciprocal hazard ratio of $x_{i}$ multiplied by a constant $z_{i}^{e}=E_{Z_{i}} z_{i}$. It is worth emphasizing that even if this is a function of a single-dimensional intensity $x_{i}$, its shape (and thus the efficient mechanism) does depend on the distribution of the penalty cost, $Z_{i}$.

One can express the payoff of member $i$ of the committee in terms of the allocation probability and the payoff of the indifferent type only. This will be asserted in the following two Lemmata. The proofs are in the Appendix.

Lemma 1. Net expected payoff of voter $i$ in an incentive compatible mechanism is

$$
N_{i}=z_{i}^{e} E_{A_{i}} \pi_{i}\left(a_{i}, 0\right)+E_{A_{i}, X_{i}} P_{a_{i}}\left(a_{i}, x_{i}\right) H_{i}\left(x_{i}\right)
$$

If $H_{i}(\cdot)$ is non-decreasing, then Equation (4) can be used in further analysis as is. However, if $H_{i}(\cdot)$ is decreasing in some places, then an additional modification will turn out to be helpful. This technique is sometimes referred to as Myerson's ironing, ${ }^{10}$ because its objective is to find a version of $H_{i}(\cdot)$ with the decreasing parts "ironed out", so that the resulting function is non-decreasing.

For every $q \in[0,1]$, define $\phi_{i}(q)=H_{i}\left(\left(F_{X_{i}}\right)^{-1}(q)\right)$, where $\left(F_{X_{i}}\right)^{-1}$ is the inverse of the marginal c.d.f. of $x_{i}$. Furthermore, let $\Phi_{i}(q)=\int_{0}^{q} \phi_{i}(s) d s$, and let

$$
\Gamma_{i}(q)=\operatorname{conv}\left(\Phi_{i}(q)\right)
$$

\footnotetext{
${ }^{10}$ See Myerson (1981) Section 6.
} 
be the convexification of function $\Phi_{i}$, and let $\gamma_{i}(q)=\Gamma_{i}^{\prime}(q)$. Obviously, all these functions are derived from the primitives of the environment, hence exogenous, and known to the designer. The next result also gives the payoff of voter $i$, and therefore is another version of Lemma 1 . However, function $H_{i}(\cdot)$, which may be decreasing, is replaced by $\gamma_{i}\left(F_{X_{i}}(\cdot)\right)$, which never is.

Lemma 2. The expected payoff of voter $i$ in an incentive compatible mechanism can be written as

$$
N_{i}=z_{i}^{e} E_{A_{i}} \pi_{i}\left(a_{i}, 0\right)-\Lambda_{i}+E_{A_{i}, X_{i}} P_{a_{i}}\left(a_{i}, x_{i}\right) \gamma_{i}\left(F_{X_{i}}\left(x_{i}\right)\right)
$$

where $\Lambda_{i} \geq 0$ is defined as

$$
\Lambda_{i}=E_{A_{i}} \int_{0}^{1} \frac{\partial P_{a_{i}}\left(a_{i}, x_{i}\right)}{\partial x_{i}}\left(\Phi_{i}\left(F_{X_{i}}\left(x_{i}\right)\right)-\Gamma_{i}\left(F_{X_{i}}\left(x_{i}\right)\right)\right) d x_{i}
$$

Lemma 2 leads to the following result. It is a counterpart of the Revenue Equivalence Theorem, known from the auction literature.

Corollary 1. For a given environment, any mechanism generating the same equilibrium expected payoff for all agents with the lowest intensity of preferences, $\pi_{i}\left(a_{i}, 0\right)$, and the same allocation function, $p_{k}(a, x)$, achieves the same welfare.

The main result follows.

Theorem 1. A mechanism that selects $B_{k}$, such that

$$
\sum_{i: a_{i}=B_{k}} \gamma_{i}\left(F_{X_{i}}\left(x_{i}\right)\right) \geq \sum_{j: a_{j}=B_{-k}} \gamma_{j}\left(F_{X_{j}}\left(x_{j}\right)\right)
$$

is efficient.

The proof is in the Appendix.

Theorem 1 looks obscure. The rest of this section outlines two special cases, one with a decreasing and one with increasing $H_{i}(\cdot)$, that lead to qualitatively different results. Broader ramifications will be discussed in the next section.

\section{Decreasing $H_{i}(\cdot)$}

It turns out that whether function $H_{i}(\cdot)$ is increasing or decreasing is of key importance to the type of the mechanism that should be selected by the efficiency-motivated designer. 
Assume that function $H_{i}(\cdot)$ is decreasing. In this case, function $\phi_{i}$ is also decreasing, and thus $\Phi_{i}$ is concave. Its convexification in Equation (5), $\Gamma_{i}$, is a straight line, and so $\gamma_{i}$ is a constant equal to $\Phi_{i}(1)$. This, in turn, is equal to the expectation $E_{X_{i}} H_{i}\left(x_{i}\right)$, which, finally, is equal to the expectation of absolute intensity $v_{i}^{e}=E_{X_{i}} x_{i} Z_{i}\left(x_{i}\right)$, where $Z_{i}\left(x_{i}\right)=\int_{0}^{\infty} z_{i} f_{Z_{i} \mid X_{i}}\left(z_{i} \mid x_{i}\right) d z_{i}$ is the expected marginal penalty cost of agent $i$, conditional on $x_{i} \cdot{ }^{11}$

Corollary 2. Suppose that $H_{i}(\cdot)$ is decreasing for every i. A mechanism that selects $B_{k}$, such that

$$
\sum_{i: a_{i}=B_{k}} v_{i}^{e} \geq \sum_{j: a_{j}=B_{-k}} v_{j}^{e}
$$

is efficient.

This condition can be assessed regardless of the reported intensities. Only reported locations matter. The mechanism gives potentially different weights to voters, but these weights $v_{1}^{e}, \ldots, v_{n}^{e}$ are known ex ante. Obviously, no penalty is needed. If values $v_{i}^{e}$ are the same for all voters, then the efficient mechanism is a classical simple majority.

Corollary 3. Suppose that $H_{i}(\cdot)$ is decreasing for every $i$. If agents' expected values $v_{i}^{e}$ are constant for every $i$, then unweighted simple majority is efficient.

Decreasing $H_{i}$ for all $i$ is a sufficient condition for the efficient mechanism to be independent of realized intensities, but it is not necessary. It is possible that functions $H_{i}$ are decreasing around the end-points of the domain and moderately increasing in the interior, and the efficient mechanism still does not use reported intensities in selecting the alternative. That is, it still is as in Corollary 2.

\section{Increasing $H_{i}(\cdot)$}

On the other hand, if $H_{i}(\cdot)$ is increasing, then convexification in Equation (5) is trivial and yields the original reciprocal hazard rate itself, $\gamma_{i}\left(F_{X_{i}}\left(x_{i}\right)\right)=$ $H_{i}\left(x_{i}\right)$. We obtain

\footnotetext{
${ }^{11}$ The fact $v_{i}^{e}=E_{X_{i}} H_{i}\left(x_{i}\right)$ is is a variant of a known result that the expectation of the reciprocal hazard rate is equal to the expectation of the random variable itself. Observe that

$$
\begin{aligned}
v_{i}^{e} & =\int_{0}^{\infty} x_{i} \int_{0}^{\infty} z_{i} f_{Z_{i} \mid X_{i}}\left(z_{i} \mid x_{i}\right) d z_{i} f_{X_{i}}\left(x_{i}\right) d x_{i}=\int_{0}^{\infty} x_{i}\left(E_{Z_{i}} z_{i} f_{i}\left(x_{i} \mid z_{i}\right)\right) d x_{i}= \\
& =-\left.x_{i} E_{Z_{i}} z_{i}\left(1-F_{i}\left(x_{i} \mid z_{i}\right)\right)\right|_{0} ^{\infty}+\int_{0}^{\infty}\left(E_{Z_{i}} z_{i}\left(1-F_{i}\left(x_{i} \mid z_{i}\right)\right)\right) d x_{i}=E_{X_{i}} H_{i}\left(x_{i}\right)
\end{aligned}
$$
}

where integration by parts was used. 
Corollary 4. Suppose $H_{i}(\cdot)$ is increasing for every i. A mechanism that selects $B_{k}$, such that

$$
\sum_{i: a_{i}=B_{k}} H_{i}\left(x_{i}\right) \geq \sum_{j: a_{j}=B_{-k}} H_{j}\left(x_{j}\right)
$$

is efficient.

That is, the mechanism asks agents to reveal not only their preference locations but also their preference intensity. There are many mechanisms that can realize the allocation function in Corollary 4. Among them, there is a version of Vickrey-Clarke-Groves mechanism, which implements outcomes in dominant strategies. We will denote this mechanism $V C G_{\text {net }}$, where the subscript indicates that the net surplus $H_{i}(\cdot)$ is used in all calculations. This mechanism works by having the voters report their types, and the mechanism then selecting alternative $k$ if and only if Inequality (8) holds. Those who supported the losing alternative are not subjected to any penalty. Those who supported the winning alternative $k$ are subjected to penalty $c_{i}(\bar{a}, \bar{x})$ defined by

$$
H_{i}\left(c_{i}(\bar{a}, \bar{x})\right)=\max \left\{H_{i}(0), \sum_{j: \bar{a}_{j}=B_{-k}} H_{j}\left(\bar{x}_{j}\right)-\sum_{\left\{j: \bar{a}_{j}=B_{k}, j \neq i\right\}} H_{j}\left(\bar{x}_{j}\right)\right\}
$$

The penalty specified in equation (9) paid by a winner is strictly positive if that voter is pivotal, that is, when reporting her true intensity instead of zero changes the outcome of voting.

Proposition 2. The $V C G_{n e t}$ mechanism has a dominant strategy, and it achieves the allocation function defined in Corollary 4.

The proof is in the Appendix.

These two special cases of decreasing and increasing $H_{i}$ do not cover all possibilities. When $H_{i}$ is not monotone, then the $V C G_{\text {net }}$ could be used where function $H_{i}(\cdot)$ is replaced by $\gamma_{i}\left(F_{X_{i}}(\cdot)\right)$.

The classical version of the Vickrey-Clarke-Groves mechanism mentioned in the introduction selects alternative $B_{k}$ if $\sum_{i: a_{i}=B_{k}} v_{i} \geq \sum_{j: a_{j}=B_{-k}} v_{j}$. Thus, it maximizes allocative efficiency, as if the penalties paid by the participants were not counted as social waste. This mechanism can be denoted $V C G_{\text {classic }}$, to distinguish it from $V C G_{\text {net. }}{ }^{12}$

\footnotetext{
${ }^{12}$ Of course, any linear combination of these two cases can be analyzed without difficulty. Suppose that only fraction $\lambda$ of the total payment is socially wasted while the remaining fraction $1-\lambda$ is transferred to some third party and hence not lost from the welfare
} 


\section{Comparative statics}

Efficient mechanisms are completely characterized by functions $H_{i}(\cdot)$. That is to say, two different environments characterized by different pairs of random variables, $\left(X_{i}, Z_{i}\right)$ and $\left(X_{i}^{\prime}, Z_{i}^{\prime}\right)$, may have the same corresponding function $H_{i}(\cdot)$, and when they do, they have the same efficient mechanism. This section examines how joint distributions of $\left(X_{i}, Z_{i}\right)$ determine the shape of $H_{i}(\cdot)$.

In the first instance, we consider the case of $X_{i}$ and $Z_{i}$ being independent. In this baseline scenario, the marginal distribution of $Z_{i}$ essentially plays no role, and the focus is on the ex ante heterogeneity of intensity of preferences, that is, the dispersion of $X_{i}$. Then, we move on to the scenarios in which $Z_{i}$ does play a non-trivial role: first, we deal with the correlation between $X_{i}$ and $Z_{i}$, and, secondly, we discuss the question of selecting a joint distribution $\left(X_{i}, Z_{i}\right)$, for an exogenously fixed marginal distribution of $V_{i}$ (which will be interpreted as a choice of a penalty type). Finally, we consider the ex post heterogeneity in efficient mechanisms.

This section assumes symmetric environments, where probability distributions are independent of $i$, that is, $F_{X}=F_{X_{i}}$ and $f_{X}=f_{X_{i}}$.

\section{Statistical independence}

Suppose that variables $X_{i}$ and $Z_{i}$ are statistically independent, and without loss of generality let $z_{i}^{e}=1$, so that $H\left(x_{i}\right)=\left(1-F_{X}\left(x_{i}\right)\right) / f_{X}\left(x_{i}\right)$.

Certainly, many distributions commonly used in examples have a decreasing reciprocal hazard rate, and therefore fall under the remit of Corollaries 2 and 3. It is sometimes claimed that this is the more likely case. ${ }^{13}$ However, as soon as we establish a link between the slope of $H(\cdot)$ and the notion of dispersion of values, this becomes an object of interpretable economic quality, which cannot be assumed away.

To illustrate the significance of the ex ante heterogeneity of individual preferences consider the following example.

perspective; or suppose that for every agent (a representative) taking part in the decision process and potentially suffering the penalties, there are $(1-\lambda) / \lambda$ identical agents freeriding on that decision (those who are represented). All conclusions, including Theorem 1, remain unaltered except that $H_{i}\left(x_{i}\right)$ is replaced by $H_{i \lambda}\left(x_{i}\right)=\lambda H_{i}\left(x_{i}\right)+(1-\lambda) x_{i} Z_{i}\left(x_{i}\right)$. This slight generalization captures the efficient mechanism when the designer considers $\lambda$ to be a good representation of welfare. For example, $\lambda=1$ leads to net efficiency and $V C G_{\text {net }}$, while $\lambda=0$ leads to allocative efficiency and $V C G_{\text {classic }}$.

${ }^{13}$ For example, McAfee and McMillan (1992) write that " $H^{\prime} \leq 0$ can be thought of as the more likely case." 
Example 1. Assume that voter's location of preferences is equally likely to be $B_{1}$ and $B_{2}$; intensity $x_{i}$ (and $v_{i}$ ) is distributed according to Weibull distribution, the same for all $i$. That is, let $F_{X}\left(x_{i}\right)=1-\exp \left(-\left(x_{i} / \mu\right)^{\eta}\right)$, and therefore $H\left(x_{i}\right)=\left(\mu^{\eta} / \eta\right) x_{i}^{1-\eta}$, where $\eta>0$ is the shape parameter, and $\mu$ is the scale parameter set up so that the mean is equal to one, $\mu=$ $1 / \Gamma(1+1 / \eta)$.

Shape parameter $\eta$ controls the ex ante heterogeneity of individual preferences. The greater this parameter, the more homogeneous within each party the intensity of preferences becomes. Conversely, if this parameter is getting closer to zero, then the more likely it is that the individual voters in a party are either almost indifferent, concentrated around zero, or if they are extreme then they are very extreme. The slope of function $H\left(x_{i}\right)$ depends on $\eta$ in a clear-cut way. If $\eta>1$, then the reciprocal hazard ratio is a decreasing function and simple majority is net efficient. If $\eta<1$, then $H(\cdot)$ is increasing, and screening for types, along the lines of Corollary 4, is net efficient.

This can be presented graphically, as in Figure 1. The curves show welfare performance (relative to simple majority) of different mechanisms for different levels of heterogeneity of preferences measured as shape parameter of Weibull distribution, $\eta$, in a committee consisting of 7 voters. The thin solid curve illustrates the level of net welfare of a mechanism achieving the best net welfare. This mechanism is simple majority if $\eta>1$ and could be $V C G_{\text {net }}$ if $\eta<1$. For comparison, the thick solid curve represents allocative welfare of a mechanism that maximizes allocative welfare, for example $V C G_{\text {classic }}$. Thus the vertical difference between the two curves represents the welfare loss when transfers cannot be used, but penalties can.

A word of caution is in order here. The above statements, that the ex ante heterogeneity of preference intensity is a key parameter, could be misunderstood, because not just any type of dispersion measure of $X_{i}$ is important. It is certainly possible to give an example of two distributions such that the first one has a greater variance and decreasing $H$, and the other one has lower variance and increasing $H$. By high heterogeneity we mean a particular relationship between central/indifferent values and tail/extreme values. This relationship is captured by the following known result, describing the relationship between the slope of the reciprocal hazard rate and the second order stochastic dominance. ${ }^{14}$

Proposition 3. If $X_{i}$ has an increasing reciprocal hazard rate, then it second order stochastically dominates $Y$ which has an exponential distribution with

\footnotetext{
${ }^{14}$ Result stated without proof. See Yoon (2011) and Shaked and Shanthikumar (1994) for a more detailed discussion of reciprocal hazard rates and the notions of dispersion.
} 


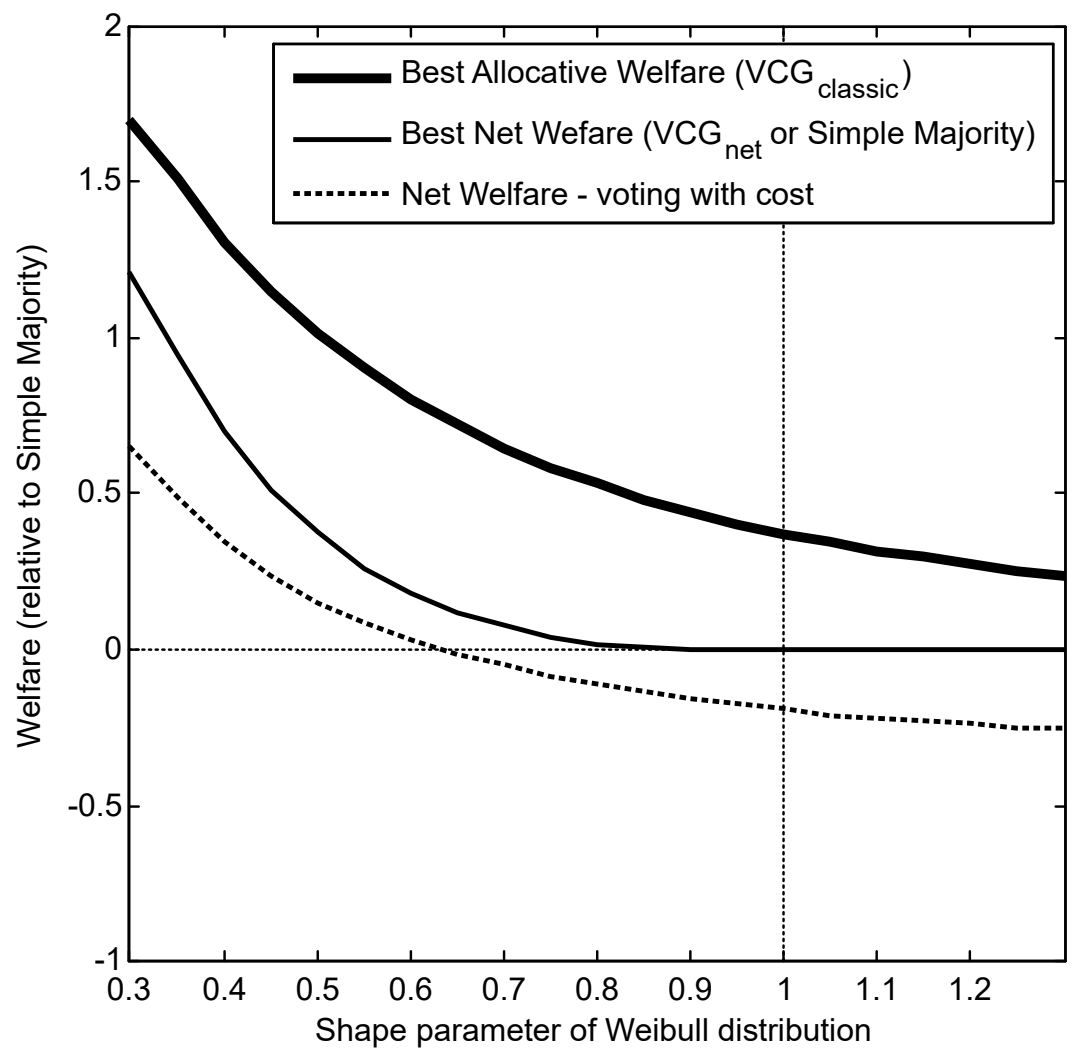

Figure 1: Solid curves: best allocative welfare and best net welfare, relative to simple majority. Dashed curve: net welfare for majority voting with participation cost. Committee $n=7$.

the same mean. If $X_{i}$ has a decreasing reciprocal hazard rate, then it is second order stochastically dominated by such $Y$.

\section{Correlation}

All the results of the previous section are completely unchanged even as the variability of $Z_{i}$ increases, for a given distribution of $X_{i}$, as long as these two random variables are independent. It is important to remember that $X_{i}$ is only the relative intensity of preferences. Agents with high cost parameters also have high absolute stakes. That is, as the variability of $Z_{i}$ increases, the variability of absolute intensity of preferences, $V_{i}=X_{i} Z_{i}$, also increases. 
The situation is more complicated if $Z_{i}$ and $X_{i}$ are not independent. Function $H\left(x_{i}\right)$ is now

$$
H\left(x_{i}\right)=\int_{0}^{\infty} z_{i} \frac{1-F\left(x_{i} \mid z_{i}\right)}{f_{X}\left(x_{i}\right)} f_{Z_{i}}\left(z_{i}\right) d z_{i} .
$$

To present a clear comparative statics, one may follow Chakravarty and Kaplan (2013) by assuming a non-stochastic relationship between $z_{i}$ and $x_{i}$. Namely, suppose that intensity of preferences is a random variable with a c.d.f. $F_{X}\left(x_{i}\right)$, but also assume that the cost parameter is determined by some monotonic function, $z_{i}=\beta\left(x_{i}\right)$, where $E \beta\left(x_{i}\right)=1$ without loss of generality. For example if $\beta$ is increasing, then the conditional c.d.f $F\left(x_{i} \mid z_{i}\right)$ is zero if $z_{i}>\beta\left(x_{i}\right)$ or one otherwise. ${ }^{15}$ In general,

$$
\begin{aligned}
H\left(x_{i}\right) & =\frac{1}{f_{X}\left(x_{i}\right)} \int_{\beta\left(x_{i}\right)}^{\infty} z_{i} f_{Z}\left(z_{i}\right) d z_{i} \\
& =\frac{1}{f_{X}\left(x_{i}\right)} \int_{x_{i}}^{\infty} \beta(s) f_{X}(s) d s
\end{aligned}
$$

For example, if $Z$ does not vary and so $\beta(\cdot)$ is a constant function, then $H(\cdot)$ becomes the usual reciprocal hazard rate.

Suppose that the association between $x_{i}$ and $z_{i}$ is positive, meaning that $\beta$ is an increasing function. How does this assumption affect the shape of $H(\cdot)$ in comparison to the conventional reciprocal hazard rate? Notice that

$$
\int_{0}^{\infty} \beta(s) f_{X}(s) d s=E \beta(s)=1
$$

and hence $f_{X}^{\beta}\left(x_{i}\right)=\beta\left(x_{i}\right) f_{X}\left(x_{i}\right)$ itself is a density function, rotated counterclockwise relative to $f_{X}\left(x_{i}\right)$. An immediate implication is that $F_{X}^{\beta}<F_{X}$ in the interior of the support, where $F_{X}^{\beta}$ is the c.d.f. associated with $f_{X}^{\beta}\left(x_{i}\right)$. This proves the first part of the following result; the second part is obtained in the same way.

Proposition 4. Assume that $x_{i}$ is in the interior of the support. If $\beta(\cdot)$ is increasing then $H\left(x_{i}\right)>\left(1-F_{X}\left(x_{i}\right)\right) / f_{X}\left(x_{i}\right)$; if $\beta(\cdot)$ is decreasing then $H\left(x_{i}\right)<\left(1-F_{X}\left(x_{i}\right)\right) / f_{X}\left(x_{i}\right)$.

The next proposition is also easy to obtain and its proof is in the Appendix.

\footnotetext{
${ }^{15}$ Such $F\left(\cdot \mid z_{i}\right)$ is not strictly increasing in the domain, as assumed in the general model in section 2. This is inconsequential.
} 
Proposition 5. Suppose that $\beta(\cdot)$ is increasing; if $\left(1-F_{X}\left(x_{i}\right)\right) / f_{X}\left(x_{i}\right)$ is increasing, then so is $H\left(x_{i}\right)$. Suppose that $\beta(\cdot)$ is decreasing; if $\left(1-F_{X}\left(x_{i}\right)\right) / f_{X}\left(x_{i}\right)$ is decreasing, then so is $H\left(x_{i}\right)$.

Both of these results give a hint of what happens when a researcher misjudges the nature of the relationship between $Z_{i}$ and $X_{i}$. The first result suggests how this error affects the magnitude of the weights used in calculating the weighted majority, while the latter indicates what is the effect on the slope of $H(\cdot)$, and thus on the question of whether it is efficient to extract information from the agents.

\section{Selecting penalty type}

It has been assumed so far that there is only one penalty type. It does make sense, however, to contemplate a range of penalties, as they may induce different behavior of the agents, and hence they may result in a different efficiency performance. For example, the designer may require money burning, idle waiting, electric shocks, squats, push-ups and a myriad other penalty types. The objective of this section is to investigate how different penalty types affect $H(\cdot)$.

What does it mean - in the logic of the current model - to replace one penalty type with a different one? From the mechanism designer's point of view, it must involve selecting a different distribution of cost parameters, $Z_{i}$. This is not all, however. One must also carefully adjust the distribution of relative intensity of preferences, $X_{i}$. To see this, consider two mechanisms. In the first mechanism, a certain penalty type is available, say push-ups, but actually no voter is ever asked to pay any amount of this penalty (for example, the voters might select an alternative by simple majority with no penalties). In the second mechanism, another penalty type is available, say squats, but no voter suffers any amount of this penalty either, and the same alternative is selected. Since in both cases the same alternative is selected and no penalty applied, the welfare achieved should be the same; our welfare measure in Equation (1) tells us that it is simply the sum of absolute values, $v_{i}$, of those voters whose alternative has been selected. This welfare level ought not to change just because a different penalty is kept in reserve, so to speak, but never actually used. However, the two scenarios use different distributions of $Z_{i}$, because that is the way in which different penalty types were defined, one distribution for push-ups and different one for squats. Consequently, the distribution of absolute values, $V_{i}=X_{i} Z_{i}$, would also be different between the scenarios, resulting in different welfare - unless, of course, the distribution of $X_{i}$ is appropriately adjusted. 
Therefore, we model the change of the penalty type by varying the joint distribution of $X_{i}$ and $Z_{i}$, such that the marginal distribution of $V_{i}$ is fixed.

To be more specific, let the c.d.f. and p.d.f. of $V_{i}$ be $F_{V}\left(v_{i}\right)$ and $f_{V}\left(v_{i}\right)$, respectively. We previously assumed for simplicity that the relative intensity and the cost parameter are linked through a non-stochastic function, $z_{i}=$ $\beta\left(x_{i}\right)$. We adopt this convention here too. This implies that the absolute and relative intensities are tied together through $v_{i}=x_{i} \beta\left(x_{i}\right)$, which can be written more concisely as a function $v_{i}=v\left(x_{i}\right)$. In this formulation, once absolute values $v_{i}$ are realized, function $v(\cdot)$ generates relative values $x_{i}$ and (through $\beta(\cdot))$ cost parameters $z_{i}$. To recapitulate: the realized absolute values come from a given distribution $F_{V}\left(v_{i}\right)$ that does not change as one replaces one penalty type with another one. Changing the penalty type is captured by changing the shape of function $v(\cdot)$.

The c.d.f and p.d.f. of $X_{i}$ can now be expressed as $F_{X}\left(x_{i}\right)=F_{V}\left(v\left(x_{i}\right)\right)$ and $f_{X}\left(x_{i}\right)=f_{V}\left(v\left(x_{i}\right)\right) v^{\prime}\left(x_{i}\right)$, respectively. Re-writing Equation (10), we finally obtain the key relationship between the penalty characterization $v(\cdot)$ and the determinant of the efficient mechanism $H(\cdot)$ used in Theorem 1 ,

$$
H\left(x_{i}\right)=\frac{1}{f_{V}\left(v\left(x_{i}\right)\right) v^{\prime}\left(x_{i}\right)} \int_{x_{i}}^{\infty} \frac{v(s)}{s} f_{V}(v(s)) v^{\prime}(s) d s
$$

To gain some intuition how a different penalty $v(\cdot)$ affects the shape of $H(\cdot)$, we investigate the following parametric example.

Example 2. Suppose that the marginal distribution of $V_{i}$ is uniform, $F_{V}\left(v_{i}\right)=$ $v_{i}$, for $v_{i} \in[0,1]$. Suppose that the designer can access a range of penalties indexed by $\alpha>0$, where the cost parameter is linked to the relative intensity through $\beta\left(x_{i}\right)=x_{i}^{\alpha-1}$, and thus the absolute and relative intensities are linked through $v\left(x_{i}\right)=x_{i}^{\alpha}$.

For instance, $\alpha=1$ brings about a case that can be naturally interpreted as a monetary penalty with a unit marginal cost, $z_{i}=\beta\left(x_{i}\right)=1$. This example generates $H\left(x_{i}\right)=1-x_{i}$. Since this function is decreasing, the efficient mechanism, when only this penalty is available, is simple majority without actually using any penalties, by Corollary 3 .

If $\alpha$ is not restricted to be one, $H\left(x_{i}\right)=x_{i}^{1-\alpha} \int_{x_{i}}^{1} s^{2(\alpha-1)} d s$. This simplifies to $H\left(x_{i}\right)=-\sqrt{x_{i}} \ln \left(x_{i}\right)$ if $\alpha=1 / 2$ and $H\left(x_{i}\right)=\left(x_{i}^{1-\alpha}-x_{i}^{\alpha}\right) /(2 \alpha-1)$ if $\alpha \neq 1 / 2$. Two qualitatively different cases emerge. If $\alpha \geq 1$ then $H(\cdot)$ is a decreasing function, implying that simple majority is efficient. If, however, $\alpha<1$, then $H(\cdot)$ is an inverted U-shaped function with $H(0)=H(1)=0$. It can be verified that the efficient $\gamma_{i}(\cdot)$ used in Theorem 1 is strictly increasing 
for low $x_{i}$, and constant (or ironed-out) for all higher $x_{i}$. The bottom-line is that simple majority is not efficient if $\alpha<1$.

Intuitively, when $\alpha<1$, the cost parameter $\beta(\cdot)$ is decreasing in $x_{i}$, and the agents who care about which alternative is selected (higher absolute and relative intensities) are the same agents who do not mind waiting (low cost parameter). It is perhaps unsurprising that the efficient mechanism involves screening if the cost of extracting information is lower when extracting information is important.

As regards which penalty the designer should select, let us contemplate a menu of penalties consisting of just two objects, $\alpha=1$ and $\alpha=1 / 2$. Notice that welfare performance of the efficient mechanism with $\alpha=1 / 2$ involves partial screening and is strictly better than simple majority for this penalty type, which is equivalent to simple majority for penalty $\alpha=1$, which, in turn, is the best mechanism for this latter penalty type. Hence, penalty $\alpha=1 / 2$ performs strictly better than $\alpha=1$.

It is obvious that this last argument is completely independent of the numerical example.

Proposition 6. If there exits a penalty type that leads to a mechanism which optimally extracts (some) information, then that penalty-mechanism pair definitely obtains higher welfare than any other mechanism not extracting information, even if not extracting information is best for some other penalty type.

This Proposition implies that investigating just one penalty type and observing that simple majority is efficient is not enough. The mechanism designer should endeavor to investigate other penalty types, because they, if associated with a suitable mechanism, may result in a higher welfare.

\section{Ex post realizations}

A concept of dispersion for vectors is needed in this section. Formally, select two vectors $x, \tilde{x} \in R^{n}$; vector $x$ is smaller in the majorization order than $\tilde{x}$, denoted $x \prec \tilde{x}$, if $\sum_{i=1}^{n} x_{i}=\sum_{i=1}^{n} \tilde{x}_{i}$ and $\sum_{i=1}^{j} x_{[i]} \leq \sum_{i=1}^{j} \tilde{x}_{[i]}$ for $j=$ $1, \ldots, n-1$, where $x_{[i]}$ denotes the $i$ th largest element of vector $x$.

Consider the following thought experiment. Compare two different realizations of preference intensities for party $k$ with the given number of supporters, denoted $x=\left\{x_{i}\right\}_{i: a_{i}=k}$ and $\tilde{x}=\left\{\tilde{x}_{i}\right\}_{i: a_{i}=k}$. Suppose that $x \prec \tilde{x}$. In other words, both realizations represented by these preference intensities are equivalent from the allocative efficiency perspective as they generate the same gross welfare for party $k$, but that the latter is more dispersed than 
the former, representing a realization that is more ex post heterogeneous. Inspecting Inequality (8), we see that the curvature of $H(\cdot)$ plays a role.

Proposition 7. Suppose that $H(\cdot)$ is increasing, and suppose that $x, \tilde{x}$ are two different realizations of preference intensities in party $k$. If $H(\cdot)$ is concave, then $x \prec \tilde{x}$ implies that $\sum_{i: a_{i}=k} H\left(x_{i}\right) \geq \sum_{i: a_{i}=k} H\left(\tilde{x}_{i}\right)$. Thus replacing $x$ with $\tilde{x}$ weakly decreases the chances of selecting alternative $k$. If $H(\cdot)$ is convex, then replacing this $x$ with this $\tilde{x}$ increases those chances.

Intuitively, less concave reciprocal hazard rate appears to be associated with a fatter tail of the distribution, and therefore with more dispersed values. To my knowledge, however, there is no established stochastic order that captures this, as was the case in the previous discussion about the sign of the slope of $H(\cdot)$.

Overall, the analysis given in this paper suggests three layers of the argument relating to ex post realization of preference intensities in an efficient mechanism. Informally, they can be stated as:

1. Is more agents in party $k$ a good news for the likelihood of selecting their party's preferred alternative? The answer is affirmative, and it does not depend on any extra conditions.

2. Suppose that the number of members in party $k$ is given. Is having members with higher preference intensity a good news for the likelihood of selecting their party's preferred alternative? The answer is affirmative, if $H$ is increasing. Intensity is irrelevant if this function is decreasing.

3. Suppose that the number of members in party $k$ is given, and they have a given total intensity, $\sum_{i: a_{i}=k} x_{i}$. Is having voters with more concentrated preference intensities (smaller in the majorization order) a good news for the likelihood of selecting their party's preferred alternative? The answer is affirmative, if, in addition to $H$ being increasing, it is a concave function. Likewise, less concentrated preference intensities is a good news, if $H$ is a convex function.

Azrieli and Kim (2014) make an observation similar to point 1 above. They say that "only the ordinal ranking of the two alternatives as reported by the agents matters for the outcome". In the current paper, the word "only" should be removed, as reporting preference intensity matters under some circumstances. But the gist is that the efficient mechanism always responds positively to the number of supporters. Another interpretation of this point invokes the Samuelson condition for optimal allocation of public goods. Namely, 
one needs to sum the benefits to all agents together in order to calculate the correct rank of each of the two alternatives. The only twist in the present paper is that those benefits are net of the costs of a nontrivial incentive provision.

The analysis of McAfee and McMillan (1992), Yoon (2011), Condorelli (2012) and Chakravarty and Kaplan (2013) underline the importance of the the monotonicity of function the reciprocal hazard rate and the role of ex ante heterogeneity of preferences, exactly along the lines of point 2 in the above list.

However, the observations made in point 3 do not arise in those studies. There is no reporting of preference intensities in Azrieli and Kim (2014) or Drexl and Kleiner (2013), and no Samuelson condition in the papers in the tradition of McAfee and McMillan (1992).

\section{Non-efficient mechanisms}

In general, various real-life mechanisms of collective choice with penalties, like those mentioned in the introduction, will not perform as well as the efficient incentive-compatible mechanism characterized in Theorem 1 . It is educational, however, to compare welfare properties of such mechanisms to some known benchmarks, taking advantage of the fact that the technique developed in this paper makes this comparison possible. One such benchmark is the efficient mechanism in Theorem 1; the other one is simple majority with no penalties.

In attempting to answer this question a major obstacle is whether it is possible to express equilibrium behavior in a given mechanism in terms of the allocation function, $p_{k}(a, x)$, and the payoff of the indifferent agent, $\pi_{i}\left(a_{i}, 0\right)$. This is not always easy or possible, but in some specific cases it can be done. When it can, then these two objects are sufficient to determine the efficiency performance of that mechanism, by Corollary 1 .

As an example, this section focuses on perhaps the simplest such mechanism - voting with a fixed participation cost. That is, agents who decide to cast their vote incur a given lump-sum cost, and the decision is made via simple majority by those who enter. For simplicity, assume that all voters are symmetric ex ante; in particular, they are equally likely to support $B_{1}$ and $B_{2}$, and they have the same distribution of preference intensity, assumed here to be the Weibull distribution from Example 1. Suppose that the cost parameter is one.

Under the assumption of symmetric strategies, agents will use a threshold strategy whereby there exist an endogenous threshold $x^{*}$ (depending on the 
participation cost) such that all relatively indifferent voters, the ones with the $x_{i}<x^{*}$, abstain, and those who care more, the ones with $x_{i} \geq x^{*}$, vote for their preferred alternative. Let $B_{k}^{*}=\left\{i: a_{i}=k, x_{i} \geq x^{*}\right\}$ denote the set of voters actively voting for $k$. It is evident that the allocation function is therefore

$$
p_{B_{1}}(a, x)= \begin{cases}1 & \text { if } \# B_{1}^{*}>\# B_{2}^{*} \\ 1 / 2 & \text { if } \# B_{1}^{*}=\# B_{2}^{*} \\ 0 & \text { if } \# B_{1}^{*}<\# B_{2}^{*}\end{cases}
$$

and the payoff of the indifferent agent is $\pi_{i}\left(a_{i}, 0\right)=0$. Clearly, $P_{a_{i}}\left(a_{i}, \cdot\right)$ is non-decreasing.

The dashed curve on Figure 1 depicts the net welfare of this mechanism, relative to simple majority, for the cost being such that the threshold ends up being $x^{*}=0.3$. Obviously, this mechanism is not net efficient, and so this curve lies below the thin solid curve representing the highest possible net welfare. However, it is interesting to know that this mechanism is better than simple majority for ex ante heterogeneity high enough ( $\eta$ low enough). ${ }^{16}$

\section{Concluding remarks}

The central presupposition of this study is that, even if monetary transfers among agents are not tolerated in social choice contexts, the designer may still be able to apply socially wasteful penalties. Correct incentives may raise the likelihood of selecting the correct alternative.

The main conclusion, relevant for designers of committee rules as well as other practitioners, can be summarized as follows: if all voters are likely to have relatively similar private stakes, then asking them to reveal only

\footnotetext{
${ }^{16}$ Voting with a participation cost is a tractable example. Another example mentioned in the introduction was that of multiplayer war of attrition. A version of this game can also be framed easily in terms of $p_{k}(a, x)$ and $\pi_{i}\left(a_{i}, 0\right)$. In particular, suppose that the supermajority rule specifies that a minority of at least $m$ voters supporting one alternative blocks the other alternative from being selected and the winning alternative is not declared for as long as both alternatives are blocked, assume that a voter stops paying the penalty by declaring exit from the game, which corresponds to "oligopoly" type of generalized war of attrition proposed by Bulow and Klemperer (1999), and finally suppose that none of the voters can observe the history of exits as the game progresses. Then the equilibrium depends on the intensity of preferences of two "pivotal" voters, that is, on the $m$ th order statistic of voter's preferences in each of two parties; the allocation function $p_{k}(a, x)$ for the symmetric equilibrium can be characterized easily. The third example mentioned in the introduction - public choice via lobbying - is more complicated because finding the allocation function requires deriving equilibrium strategy, which appears to be straightforward in principle, but intractable with paper and pencil.
} 
ordinal preferences is efficient. If, however, there is likely to be a lot of fairly indifferent voters and a few extreme ones, then the designer ought to consider more complicated rules that, in particular, involve creating incentives to reveal how high the agents' stakes in the decision problem are. This analysis can be conducted even if voters' cost parameters are unknown to the designer and other agents; particular stochastic properties of these cost parameters, such as correlation with the intensity of preferences, are important.

The efficient voting rules have properties that are familiar from the auction literature, but may seem quite exotic in the context of collective choice. I am not familiar with any real-life examples of committees using penalties that resemble the efficient mechanism outlined in the current paper. However, the main conclusion of the previous paragraph may still be valid, even if the committee designer is allowed to use more natural procedures. That is, there may be natural-looking mechanisms that are more efficient than simple majority.

Some assumptions of the current model are questionable in some important ways. Firstly, there are only two alternatives. Consequently, the assumption that intensity of preferences can be viewed as a single-dimensional type is acceptable. If there were three or more alternatives (like, for example, in Gershkov et al. (2014)), then it would not at all be clear how to capture private information in such a way that is both tractable and economically relevant. ${ }^{17}$ Secondly, private information is assumed to be statistically independent across voters. As is well known in auction literature, if types are correlated, or affiliated, then a counterpart of Proposition 1 does not exist (in voting, correlated types are studied, for example, by Schmitz and Tröger (2012)). Thirdly, it seems that the main issue that the committee design should address in practice is the one of collective information acquisition (like, for example, in Persico (2004)). Future work should investigate how introducing penalties into a collective choice problem would affect the efficient mechanism in these cases.

\footnotetext{
${ }^{17}$ One restriction could be that the payoff is of the Hamming distance type. That is, voter $i$ obtains payoff $v_{i}$ if her preferred alternative is selected, and zero otherwise, no matter which of many other alternatives occurs. Under this assumption, Theorem 1 immediately generalizes, in the sense that the selected alternative, $k$, should be the one that has the highest "score" $\sum_{i: a_{i}=B_{k}} \gamma_{i}\left(F_{X_{i}}\left(x_{i}\right)\right)$. In short, the efficient mechanism becomes a weighted first-past-the-post system, in which each voter's weight is in principle type-dependent. However, the Hamming distance is very restrictive: all alternatives that are not first-best for an individual are equally bad. Therefore, any notion of a compromise with regards to the location of the eventual policy is meaningless.
} 


\section{Proofs}

\section{Proof of Proposition 1}

Necessity. Suppose that the mechanism induces truth telling.

1. $P_{a_{i}}\left(a_{i}, \cdot, z_{i}\right)$ is non-decreasing.

Truth telling implies that (where the scaling factor $z_{i}$ in front is not included because it does not affect anything)

$$
\begin{aligned}
\pi_{i}\left(r_{i}\right) & \geq \tilde{\pi}_{i}\left(\bar{r}_{i}, r_{i}\right)=x_{i} P_{a_{i}}\left(\bar{r}_{i}\right)-C_{i}\left(\bar{r}_{i}\right) \\
& =\left(x_{i}-\bar{x}_{i}\right) P_{a_{i}}\left(\bar{r}_{i}\right)+\bar{x}_{i} P_{a_{i}}\left(\bar{r}_{i}\right)-C_{i}\left(\bar{r}_{i}\right) \\
& =\left(x_{i}-\bar{x}_{i}\right) P_{a_{i}}\left(\bar{r}_{i}\right)+\pi_{i}\left(\bar{r}_{i}\right)
\end{aligned}
$$

Hence, if $x_{i}>\bar{x}_{i}$ we obtain the inequality on the left in

$$
P_{a_{i}}\left(\bar{r}_{i}\right) \leq \frac{\pi_{i}\left(r_{i}\right)-\pi_{i}\left(\bar{r}_{i}\right)}{x_{i}-\bar{x}_{i}} \leq P_{a_{i}}\left(r_{i}\right) .
$$

Similarly, using $\pi_{i}\left(\bar{r}_{i}\right) \geq \tilde{\pi}_{i}\left(r_{i}, \bar{r}_{i}\right)$ we obtain the inequality on the right. Since this is true for arbitrary reports of location and cost parameter, even true ones, $\bar{a}_{i}=a_{i}$ and $\bar{z}_{i}=z_{i}$, the function $P_{a_{i}}\left(a_{i}, \cdot, z_{i}\right)$ is nondecreasing.

2. $\frac{\partial}{\partial x_{i}} \pi_{i}\left(a_{i}, x_{i}, z_{i}\right)=P_{a_{i}}\left(a_{i}, x_{i}, z_{i}\right)$

A non-decreasing function $P_{a_{i}}\left(a_{i}, \cdot, z_{i}\right)$ is differentiable almost everywhere, hence continuous. We obtain the result by taking a limit $\bar{x}_{i} \rightarrow$ $x_{i}$.

3. $P_{a_{i}}\left(a_{i}, 0, z_{i}\right) \geq P_{a_{i}}\left(-a_{i}, 0, z_{i}\right)$ and $C_{i}\left(a_{i}, 0, z_{i}\right)=C_{i}\left(-a_{i}, 0, z_{i}\right)$

Suppose that $C_{i}\left(k, 0, z_{i}\right)>C_{i}\left(-k, 0, z_{i}\right)$. Then a voter whose true type is $\left(k, 0, z_{i}\right)$ would have incentives to misreport the location of her preferences, as their payoff would be more negative if they reported $k$. Hence $C_{i}\left(a_{i}, 0, z_{i}\right)=C_{i}\left(-a_{i}, 0, z_{i}\right)$. Finally, consider a voter whose preferences are directed towards $a_{i}$. Telling the truth must be better than stating a different party and misreporting the intensity as zero:

$$
x_{i} P_{a_{i}}\left(a_{i}, x_{i}, z_{i}\right)-C_{i}\left(a_{i}, x_{i}, z_{i}\right) \geq x_{i} P_{a_{i}}\left(-a_{i}, 0, z_{i}\right)-C_{i}\left(-a_{i}, 0, z_{i}\right)
$$

Since penalty $C_{i}\left(a_{i}, \cdot, z_{i}\right)$ is non-decreasing, ${ }^{18}$ we have $C_{i}\left(a_{i}, x_{i}, z_{i}\right) \geq$ $C_{i}\left(a_{i}, 0, z_{i}\right)=C_{i}\left(-a_{i}, 0, z_{i}\right)$, and thus Inequality (11) becomes

$$
P_{a_{i}}\left(a_{i}, x_{i}, z_{i}\right) \geq P_{a_{i}}\left(-a_{i}, 0, z_{i}\right)
$$

\footnotetext{
${ }^{18}$ This can be seen by taking the derivative of both sides in Equation (3) with respect to $x_{i}$ and using already established condition 2 .
} 
This is true for any $x_{i}$ and so it must be true for $x_{i}=0$.

4. $\pi_{i}\left(a_{i}, x_{i}, \cdot\right)$ and $P_{a_{i}}\left(a_{i}, x_{i}, \cdot\right)$ are constant.

Firstly, $\tilde{\pi}_{i}\left(a_{i}, x_{i}, \bar{z}_{i}, a_{i}, x_{i}, z_{i}\right)$ is independent of $z_{i}$ by definition in Equation (2). Secondly, it is independent of the report $\bar{z}_{i}$ too. (To see this, fix $a_{i}, x_{i}$ and note that the set of reports $\bar{z}_{i}$ that maximize the expected payoff $z_{i} \tilde{\pi}_{i}\left(a_{i}, x_{i}, \bar{z}_{i}, a_{i}, x_{i}, z_{i}\right)$ does not depend on $z_{i}$, by previous point. If a certain report does not belong to this set then there is an incentive to lie, which cannot be a part of equilibrium. Hence all reports are maximizers and the function must be constant). The claim comes from the fact that $\pi_{i}\left(a_{i}, x_{i}, z_{i}\right)=\tilde{\pi}_{i}\left(a_{i}, x_{i}, z_{i}, a_{i}, x_{i}, z_{i}\right)$, and that the right-hand side is independent of $z_{i}$.

Since $\pi_{i}\left(a_{i}, x_{i}, z_{i}\right)$ is constant over $z_{i}$ and $\frac{\partial}{\partial x_{i}} \pi_{i}\left(a_{i}, x_{i}, z_{i}\right)=P_{a_{i}}\left(a_{i}, x_{i}, z_{i}\right)$, it must be that $P_{a_{i}}\left(a_{i}, x_{i}, z_{i}\right)$ is constant over $z_{i}$.

Sufficiency. Suppose that conditions 1-4 hold.

1. Reporting true location $a_{i}$, but misreporting $x_{i}$ can never improve the expected payoff (regardless of the report $\bar{z}_{i}$ ).

Consider any $\bar{x}_{i}>x_{i}$ and any $\bar{z}_{i}$. Then the condition $\frac{\partial}{\partial s} \pi_{i}\left(a_{i}, s, \bar{z}_{i}\right)=$ $P_{a_{i}}\left(a_{i}, s, \bar{z}_{i}\right)$ implies that

$$
\pi_{i}\left(a_{i}, \bar{x}_{i}, \bar{z}_{i}\right)-\pi_{i}\left(a_{i}, x_{i}, \bar{z}_{i}\right)=\int_{x_{i}}^{\bar{x}_{i}} P_{a_{i}}\left(a_{i}, s, \bar{z}_{i}\right) d s
$$

Since $P_{a_{i}}\left(a_{i}, \cdot, \bar{z}_{i}\right)$ is non-decreasing, we have

$$
\int_{x_{i}}^{\bar{x}_{i}} P_{a_{i}}\left(a_{i}, s, \bar{z}_{i}\right) d s \leq\left(\bar{x}_{i}-x_{i}\right) P_{a_{i}}\left(a_{i}, \bar{x}_{i}, \bar{z}_{i}\right)
$$

and therefore together

$$
\pi_{i}\left(a_{i}, \bar{x}_{i}, \bar{z}_{i}\right)-\pi_{i}\left(a_{i}, x_{i}, \bar{z}_{i}\right) \leq\left(\bar{x}_{i}-x_{i}\right) P_{a_{i}}\left(a_{i}, \bar{x}_{i}, \bar{z}_{i}\right)
$$

Substituting in $\pi_{i}\left(a_{i}, \bar{x}_{i}, \bar{z}_{i}\right)=\bar{x}_{i} P_{a_{i}}\left(a_{i}, \bar{x}_{i}, \bar{z}_{i}\right)-C_{i}\left(a_{i}, \bar{x}_{i}, \bar{z}_{i}\right)$ implies

$$
z_{i}\left(x_{i} P_{a_{i}}\left(a_{i}, \bar{x}_{i}, \bar{z}_{i}\right)-C_{i}\left(a_{i}, \bar{x}_{i}, \bar{z}_{i}\right)\right) \leq z_{i} \pi_{i}\left(a_{i}, x_{i}, \bar{z}_{i}\right)
$$

One can show a similar inequality for $\bar{x}_{i}<x_{i}$. This means that reporting true location $a_{i}$, but misreporting $x_{i}$ can never improve the expected payoff (regardless of the report of $\bar{z}_{i}$ ). 
2. Misreporting the location of support does not increase payoff.

Observe that $P_{a_{i}}\left(-a_{i}, \cdot, z_{i}\right)$ is non-increasing. This follows from the fact that probabilities add up to one, that is, for any $a_{i}, x_{i}, z_{i}$

$$
1=P_{a_{i}}\left(a_{i}, x_{i}, z_{i}\right)+P_{-a_{i}}\left(a_{i}, x_{i}, z_{i}\right)
$$

where $-a_{i}$ in the subscript indicates the alternative that voter $i$ does not prefer. Thus, if $P_{a_{i}}\left(a_{i}, \cdot, z_{i}\right)$ is non-decreasing by condition 1 , then $P_{-a_{i}}\left(a_{i}, \cdot, z_{i}\right)$ is non-increasing, so is $P_{a_{i}}\left(-a_{i}, \cdot, z_{i}\right)$. As noted in footnote 18 , function $C_{i}\left(a_{i}, \cdot, z_{i}\right)$ is non-decreasing, so the function

$$
\tilde{\pi}_{i}\left(-a_{i}, \cdot, \bar{z}_{i}, r_{i}\right)=x_{i} P_{a_{i}}\left(-a_{i}, \cdot, \bar{z}_{i}\right)-C_{i}\left(-a_{i}, \cdot, \bar{z}_{i}\right)
$$

is non-increasing.

In other words, if a voter masquerades herself as a member of a different party, then her payoff will be at least as high as if she also misrepresented her intensity as zero. This is the first inequality of the chain below. The second and third lines follow from condition 3, and the final line comes from step one above.

$$
\begin{aligned}
\tilde{\pi}_{i}\left(-a_{i}, \bar{x}_{i}, \bar{z}_{i}, r_{i}\right) & \leq x_{i} P_{a_{i}}\left(-a_{i}, 0, \bar{z}_{i}\right)-C_{i}\left(-a_{i}, 0, \bar{z}_{i}\right) \\
& =x_{i} P_{a_{i}}\left(-a_{i}, 0, \bar{z}_{i}\right)-C_{i}\left(a_{i}, 0, \bar{z}_{i}\right) \\
& \leq x_{i} P_{a_{i}}\left(a_{i}, 0, \bar{z}_{i}\right)-C_{i}\left(a_{i}, 0, \bar{z}_{i}\right) \\
& \leq x_{i} P_{a_{i}}\left(a_{i}, x_{i}, \bar{z}_{i}\right)-C_{i}\left(a_{i}, x_{i}, \bar{z}_{i}\right)=\tilde{\pi}_{i}\left(a_{i}, x_{i}, \bar{z}_{i}, r_{i}\right)
\end{aligned}
$$

This means that $z_{i} \tilde{\pi}_{i}\left(-a_{i}, \bar{x}_{i}, \bar{z}_{i}, r_{i}\right) \leq z_{i} \tilde{\pi}_{i}\left(a_{i}, x_{i}, \bar{z}_{i}, r_{i}\right)$, or that misreporting a location is never better than stating the location and value correctly (regardless of report $\bar{z}_{i}$ ).

3. Finally, note that if $\pi_{i}\left(\bar{a}_{i}, \bar{x}_{i}, \cdot\right)$ and $P_{a_{i}}\left(\bar{a}_{i}, \bar{x}_{i}, \cdot\right)$ are constant then so is $C_{i}\left(\bar{a}_{i}, \bar{x}_{i}, \cdot\right)$; misreporting $z_{i}$ does not improve payoff.

\section{Proof of Lemma 1}

Since the payoff of voter $i$ in an incentive compatible mechanism is $z_{i} \pi_{i}\left(a_{i}, x_{i}\right)$ the expected payoff is

$$
N_{i}=E_{A_{i}, Z_{i}} \int_{0}^{\infty} z_{i} \pi_{i}\left(a_{i}, x_{i}\right) f_{i}\left(x_{i} \mid z_{i}\right) d x_{i}
$$

Integrate by parts

$$
N_{i}=-\left.E_{A_{i}, Z_{i}} z_{i} \pi_{i}\left(a_{i}, x_{i}\right)\left(1-F_{i}\left(x_{i} \mid z_{i}\right)\right)\right|_{0} ^{\infty}+E_{A_{i}, Z_{i}} \int_{0}^{\infty} \frac{\partial \pi_{i}\left(a_{i}, x_{i}\right)}{\partial x_{i}} z_{i}\left(1-F_{i}\left(x_{i} \mid z_{i}\right)\right) d x_{i}
$$


Use condition 2 of Proposition 1

$$
\begin{aligned}
N_{i} & =E_{A_{i}, Z_{i}} z_{i} \pi_{i}\left(a_{i}, 0\right)+E_{A_{i}, Z_{i}} \int_{0}^{\infty} P_{a_{i}}\left(a_{i}, x_{i}\right) z_{i} \frac{1-F_{i}\left(x_{i} \mid z_{i}\right)}{f_{X_{i}}\left(x_{i}\right)} f_{X_{i}}\left(x_{i}\right) d x_{i} \\
& =z_{i}^{e} E_{A_{i}} \pi_{i}\left(a_{i}, 0\right)+E_{A_{i}} \int_{0}^{\infty} P_{a_{i}}\left(a_{i}, x_{i}\right)\left(E_{Z_{i}} z_{i} \frac{1-F_{i}\left(x_{i} \mid z_{i}\right)}{f_{X_{i}}\left(x_{i}\right)}\right) f_{X_{i}}\left(x_{i}\right) d x_{i}
\end{aligned}
$$

Substitute $H_{i}\left(x_{i}\right)=E_{Z_{i}} z_{i} \frac{1-F_{i}\left(x_{i} \mid z_{i}\right)}{f_{X_{i}}\left(x_{i}\right)}$

$$
N_{i}=z_{i}^{e} E_{A_{i}} \pi_{i}\left(a_{i}, 0\right)+E_{A_{i}, X_{i}} P_{a_{i}}\left(a_{i}, x_{i}\right) H_{i}\left(x_{i}\right)
$$

\section{Proof of Lemma 2}

By adding and subtracting $E_{A_{i}, X_{i}} P_{a_{i}}\left(a_{i}, x_{i}\right) \gamma_{i}\left(F_{X_{i}}\left(x_{i}\right)\right)$, payoff in Equation (4) can be written as

$$
\begin{aligned}
N_{i}= & z_{i}^{e} E_{A_{i}} \pi_{i}\left(a_{i}, 0\right)+E_{A_{i}, X_{i}} P_{a_{i}}\left(a_{i}, x_{i}\right) \gamma_{i}\left(F_{X_{i}}\left(x_{i}\right)\right) \\
& +E_{A_{i}, X_{i}} P_{a_{i}}\left(a_{i}, x_{i}\right)\left(\phi_{i}\left(F_{X_{i}}\left(x_{i}\right)\right)-\gamma_{i}\left(F_{X_{i}}\left(x_{i}\right)\right)\right)
\end{aligned}
$$

The last component on the right can be integrated by parts and written as

$$
\begin{aligned}
& \left.E_{A_{i}} P_{a_{i}}\left(a_{i}, x_{i}\right)\left(\Phi_{i}\left(F_{X_{i}}\left(x_{i}\right)\right)-\Gamma_{i}\left(F_{X_{i}}\left(x_{i}\right)\right)\right)\right|_{0} ^{1} \\
& -E_{A_{i}} \int_{0}^{1} \frac{\partial P_{a_{i}}\left(a_{i}, x_{i}\right)}{\partial x_{i}}\left(\Phi_{i}\left(F_{X_{i}}\left(x_{i}\right)\right)-\Gamma_{i}\left(F_{X_{i}}\left(x_{i}\right)\right)\right) d x_{i}
\end{aligned}
$$

Note that because of the convexification, we have $\Phi_{i}(0)=\Gamma_{i}(0)$ and $\Phi_{i}(1)=$ $\Gamma_{i}(1)$ at the end points; so the first term is equal to zero. This establishes payoff in Equation (6), and the definition of $\Lambda_{i}$.

To show that $\Lambda_{i}$ is non-negative, notice that $\partial P_{a_{i}}\left(a_{i}, x_{i}\right) / \partial x_{i} \geq 0$, and, because of convexification, we have $\Phi_{i}(q) \geq \Gamma_{i}(q)$ in the entire domain.

\section{Proof of Theorem 1}

By Lemma 2, total welfare is bounded

$$
\sum_{i} N_{i} \leq E_{A, X} \sum_{i} p_{a_{i}}(a, x) \gamma_{i}\left(F_{X_{i}}\left(x_{i}\right)\right)
$$

The proof is conducted in two steps. Firstly, we will find $p_{a_{i}}(a, x)$ that maximizes this upper bound. Secondly, we will observe that this optimal function also guarantees that the upper bound is reached with equality, and thus it also maximizes total welfare itself. 
Step 1. Take the sum under the expectation and write it as two separate terms for individuals who support $B_{1}$ and $B_{2}$, respectively,

$$
\begin{aligned}
\sum_{i} N_{i} & \leq E_{A, X}\left(\sum_{i: a_{i}=B_{1}} p_{B_{1}}\left(a_{i}, a_{-i}, x\right) \gamma_{i}\left(F_{X_{i}}\left(x_{i}\right)\right)+\sum_{j: a_{j}=B_{2}} p_{B_{2}}\left(a_{j}, a_{-j}, x\right) \gamma_{j}\left(F_{X_{j}}\left(x_{j}\right)\right)\right) \\
& =E_{A, X}\left(p_{B_{1}}(a, x) \sum_{i: a_{i}=B_{1}} \gamma_{i}\left(F_{X_{i}}\left(x_{i}\right)\right)+p_{B_{2}}(a, x) \sum_{j: a_{j}=B_{2}} \gamma_{j}\left(F_{X_{j}}\left(x_{j}\right)\right)\right)
\end{aligned}
$$

Notice that $p_{B_{2}}(a, x)=1-p_{B_{1}}(a, x)$, and hence

$\sum_{i} N_{i} \leq E_{A, X}\left(p_{B_{1}}(a, x)\left(\sum_{i: a_{i}=B_{1}} \gamma_{i}\left(F_{X_{i}}\left(x_{i}\right)\right)-\sum_{j: a_{j}=B_{2}} \gamma_{j}\left(F_{X_{j}}\left(x_{j}\right)\right)\right)+\sum_{j: a_{j}=B_{2}} \gamma_{j}\left(F_{X_{j}}\left(x_{j}\right)\right)\right)$

The bound is maximized if the following rule is applied: Select $B_{k}$ with probability 1 if

$$
\sum_{i: a_{i}=B_{k}} \gamma_{i}\left(F_{X_{i}}\left(x_{i}\right)\right) \geq \sum_{j: a_{j}=B_{-k}} \gamma_{j}\left(F_{X_{j}}\left(x_{j}\right)\right)
$$

This establishes a candidate solution $p_{k}(a, x)$. Notice that this function generates an non-decreasing $P_{k}\left(a_{i}, x_{i}\right)$; this is true because $\gamma_{i}$ is monotonically increasing by construction.

Step 2. The last thing is to show that the proposed mechanism achieves this (maximal) bound. That is, we want to show that if the candidate solution is used then $\pi_{i}\left(a_{i}, 0\right)=0$ and $\Lambda_{i}=0$ for all individuals.

First, notice that the indifferent type is not expected to pay anything, hence $\pi_{i}\left(a_{i}, 0\right)=0$.

Secondly, we show that if there exists an $x_{i}$ such that $\Phi_{i}\left(F_{X_{i}}\left(x_{i}\right)\right)>$ $\Gamma_{i}\left(F_{X_{i}}\left(x_{i}\right)\right)$, then $\partial P_{a_{i}}\left(a_{i}, x_{i}\right) / \partial x_{i}=0$ for all $a_{i}$, and hence the second part of $\Lambda_{i}$ is equal to zero too, proving the claim. Suppose that there is an $x_{i}$ for which $\Phi_{i}\left(F_{X_{i}}\left(x_{i}\right)\right)>\Gamma_{i}\left(F_{X_{i}}\left(x_{i}\right)\right)$. But in this situation, the convexification $\Gamma_{i}$ is linear, and hence its derivative, $\gamma_{i}$, is constant in its neighborhood. If this is the case, then a small change of such $x_{i}$ in condition $\sum_{i: a_{i}=B_{1}} \gamma_{i}\left(F_{X_{i}}\left(x_{i}\right)\right) \geq$ $\sum_{j: a_{j}=B_{2}} \gamma_{j}\left(F_{X_{j}}\left(x_{j}\right)\right)$ will not change the allocation probability $p_{k}(a, x)$ and so $\partial P_{a_{i}}\left(a_{i}, x_{i}\right) / \partial x_{i}=0$.

\section{Proof of Proposition 2}

The mechanism does not make any use of report $\bar{z}_{i}$ so misreporting it does not improve the payoff regardless of the behavior of other voters. 
Consider now reporting true location but misreporting intensity. Notice that the penalty of voter $i$ conditional on winning, $c_{i}(\bar{a}, \bar{x})$, is independent of her report. This is because the righ-hand side of Equation (9) does not depend on the report of voter $i$ 's intensity. So, the only element of the outcome that voter $i$ can affect by choosing different reports is her winninglosing status.

Suppose that other voters vote such that

$$
H_{i}(0) \leq \sum_{j: \bar{a}_{i}=B_{-k}} H_{j}\left(\bar{x}_{j}\right)-\sum_{\left\{j: \bar{a}_{i}=B_{k}, j \neq i\right\}} H_{j}\left(\bar{x}_{j}\right)
$$

The penalty is set so that

$$
H_{i}\left(c_{i}(\bar{a}, \bar{x})\right)+\sum_{\left\{j: \bar{a}_{i}=B_{k}, j \neq i\right\}} H_{j}\left(\bar{x}_{j}\right)=\sum_{j: \bar{a}_{i}=B_{-k}} H_{j}\left(\bar{x}_{j}\right)
$$

That is, if $x_{i} \geq c_{i}(\bar{a}, \bar{x})$ then reporting truthfully will guarantee that the alternative preferred by voter $i$ will be selected, because $H_{i}(\cdot)$ is assumed to be increasing. However, since in this case the individual payoff, $z_{i}\left(x_{i}-c_{i}(\bar{a}, \bar{x})\right)$, is positive too, voter $i$ wants his alternative to be selected at this price. Alternatively, if $x_{i}<c_{i}(\bar{a}, \bar{x})$ then reporting truthfully will guarantee that the alternative preferred by voter $i$ will not be selected. However, since in this case the individual payoff is negative, voter does not want to change this situation.

If on the other hand

$$
H_{i}(0)>\sum_{j: \bar{a}_{i}=B_{-k}} H_{j}\left(\bar{x}_{j}\right)-\sum_{\left\{j: \bar{a}_{i}=B_{k}, j \neq i\right\}} H_{j}\left(\bar{x}_{j}\right)
$$

then penalty is zero $c_{i}(\bar{a}, \bar{x})=0$. If voter $i$ reports truthfully, then the condition

$$
H_{i}\left(x_{i}\right)>\sum_{j: \bar{a}_{i}=B_{-k}} H_{j}\left(\bar{x}_{j}\right)-\sum_{\left\{j: \bar{a}_{i}=B_{k}, j \neq i\right\}} H_{j}\left(\bar{x}_{j}\right)
$$

holds and his alternative is selected. Hence, regardless of what other voters do, voter $i$ has no incentives to misreport her intensity.

\section{Proof of Proposition 5}

Observe that if $\left(1-F_{X}(x)\right) / f_{X}(x)$ is increasing then $-1 / \int_{x}^{\infty} f_{X}(s) d s>$ $\left(f_{X}^{\prime} / f_{X}^{2}\right)$, simply by taking the derivative.

Now, suppose that $\beta$ is increasing. Thus

$$
\int_{x}^{\infty} \beta(x) f_{X}(s) d s<\int_{x}^{\infty} \beta(s) f_{X}(s) d s
$$


or

$$
-\frac{\beta(x)}{\int_{x}^{\infty} \beta(s) f_{X}(s) d s}>-\frac{1}{\int_{x}^{\infty} f_{X}(s) d s}
$$

This implies that

$$
-\frac{\beta(x)}{\int_{x}^{\infty} \beta(s) f_{X}(s) d s}>\left(f_{X}^{\prime} / f_{X}^{2}\right)
$$

But this implies that $H^{\prime}(x)=-\beta(x)-\left(f_{X}^{\prime} / f_{X}^{2}\right) \int_{x}^{\infty} \beta(s) f_{X}(s) d s$ is positive. The second part of the proposition can be obtained in a similar way.

\section{Literature}

Apesteguia, J., Ballester M.A. , Ferrer R., (2011), On the Justice of Decision Rules, Review of Economic Studies, Vol. 78, No. 1, pp. 1-16.

Armstrong, Mark, (1996), Multiproduct Nonlinear Pricing, Econometrica, Vol. 64, No. 1, pp. 51-75.

Arrow K. J. (1979), The property rights doctrine and demand revelvation under incomplete information. In: Boskin M. (ed) Economics and human wealth. Academic Press, New York.

Azrieli Y. and S. Kim, (2014) Pareto Efficiency And Weighted Majority Rules, International Economic Review Vol. 55, No. 4, pp. 1067-1088.

Bulow, J., and P. D. Klemperer (1999): "The Generalized War of Attrition," American Economic Review, Vol. 89, No. 1, pp. 175-89.

Clarke, E., (1971), Multipart Pricing of Public Goods, Public Choice, Vol. 11, No. 1, pp. 17-33.

Casella, Alessandra, (2005), Storable votes, Games and Economic Behavior, Vol. 51, No. 2, pp. 391-419.

Casella, Alessandra, Andrew Gelman and Thomas R. Palfrey, (2006), An experimental study of storable votes, Games and Economic Behavior, Vol. 57, No. 1, pp. 123-154.

Casella, Alessandra and Andrew Gelman, (2008), A simple scheme to improve the efficiency of referenda, Journal of Public Economics, Vol. 92, No. 10-11, pp. 2240-2261.

Chakravarty, S., Kaplan T.R., (2013), Optimal allocation without transfer payments, Games and Economic Behavior, Vol. 77, No. 1, pp. 1-20. 
Condorelli, D., (2012), What money can't buy: Efficient mechanism design with costly signals, Games and Economic Behavior, Vol. 75, No. 2, pp. 613-624.

d'Aspremont, Claude and Gerard-Varet, Louis-Andre, (1979), Incentives and incomplete information, Journal of Public Economics, Vol. 11, No. 1, pp. 25-45.

Drexl M., and Kleiner, A., (2013), Why voting, A Welfare Analysis, unpublished manuscript.

Gershkov, Alex, Moldovanu, Benny and Shi, Xianwen, (2014), Optimal Voting Rules, unpublished manuscript.

Goeree, Jacob K. and Jingjing Zhang, (2013), Electoral Engineering: One Man, One Vote Bid, unpublished manuscript.

Groves, Theodore, (1973), Incentives in Teams, Econometrica, Vol. 41, No. 4, pp. 617-631.

Groves, Theodore and John O. Ledyard, (1977), Some Limitations of Demand Revealing Processes, Public Choice, Vol. 29, No. 2, pp. 107-124.

Hartline, Jason D. and Roughgarden, Tim, (2008), Optimal Mechanism Design and Money Burning, in: STOC'08 Conference Proceedings.

Jackson, Matthew O. and Sonnenschein, Hugo F, (2007), Overcoming Incentive Constraints by Linking Decisions, Econometrica, Vol. 75, No. 1, pp. 241-257.

Kwiek M. (2014), Conclave, European Economic Review, Vol. 70, Issue C, pp. 258-275.

Kwiek, M. and Zhang, Q., (2013), Majority Rule and Mechanism Design, unpublished manuscript.

Kwiek, M. Marreiros, Helia, and Vlassopoulos, Michael, (2016), An Experimental Study of Voting with Costly Delay, Economic Letters, Vol. 160, Issue C, pp. 23-26.

Ledyard, John O. and Thomas R. Palfrey, (1994), Voting and Lottery Drafts as Efficient Public Goods Mechanisms, Review of Economic Studies, Vol. 61, No. 2, pp. 327-355. 
Ledyard, John O. and Thomas R. Palfrey, (1999), A Characterization of Interim Efficiency with Public Goods, Econometrica, Vol. 67, No 2, pp. $435-448$.

Ledyard, John O. and Thomas R. Palfrey, (2002), The approximation of efficient public good mechanisms by simple voting schemes, Journal of Public Economics, Vol. 83, No. 2, pp. 153-171.

McAfee, R.P., McMillan, J. (1992), Bidding rings, American Economic Review, Vol. 82, No. 3, pp. 579-599.

Myerson, R.B., (1981), Optimal auction design, Mathematics of Operations Research, Vol. 6, No. 1, pp. 58-73.

Mas-Colell, Andreu, Michael D. Whinston and Jerry R. Green, (1995), Microeconomic Theory, Oxford University Press.

May, Kenneth O., (1952), A Set of Independent, Necessary and Sufficient Conditions for Simple Majority Decision, Econometrica, Vol. 20, pp. 680-684.

Persico, N. (2004), Committee Design with Endogenous Information, Review of Economic Studies, Vol. 71, No. 1, pp. 165-94.

Ponsati, Clara and Sakovics, Jozsef, (1996), Multiperson Bargaining over Two Alternatives, Games and Economic Behavior, Elsevier, Vol. 12, No. 2, pp. 226-244.

Rae, Douglas W. (1969), Decision-Rules and Individual Values in Constitutional Choice, The American Political Science Review, Vol. 63, No. 1, pp. $40-56$.

Rob, Rafael, (1982), Asymptotic efficiency of the demand revealing mechanism, Journal of Economic Theory, Vol. 28, No. 2, pp. 207-220.

Samuelson, Paul A., (1954), The Pure Theory of Public Expenditure, The Review of Economics and Statistics, Vol. 36, No. 4, pp. 387-389.

Schmitz, Patrick W. and Tröger, Thomas, (2012), The (sub-)optimality of the majority rule, Games and Economic Behavior, Vol. 74, No. 2, pp. 651-665.

Shaked, Moshe and Shanthikumar, J. George, (1994), Stochastic orders and their applications, Boston Academic Press. 
Tideman, T. Nicolaus and Gordon Tullock (1976), A New and Superior Process for Making Social Choices, Journal of Political Economy, Vol. 84, No. 6, pp. 1145-1159.

Yoon, K. (2011), Optimal mechanism design when both allocative inefficiency and expenditure inefficiency matter, Journal of Mathematical Economics, Vol. 47, No. 6, pp. 670-676.

Vickrey, W. (1961), Counterspeculation, Auctions, and Competitive Sealed Tenders, Journal of Finance, Vol. 16, No. 1, 8-37. 